\title{
Computational Technology for Solving Nonconvex Optimal Control Problems for Power Systems
}

\author{
Alexander Yu. Gornov ${ }^{1}$, Tatiana S. Zarodnyuk ${ }^{2}$ \\ ${ }^{1}$ Matrosov Institute for System Dynamics and Control Theory of Siberian Branch of the Russian Academy of Sciences \\ Laboratory of Optimal Control, \\ Lermontov str., 134 \\ Irkutsk, Russia \\ E-mail: gornov@icc.ru \\ ${ }^{2}$ Matrosov Institute for System Dynamics and Control Theory of Siberian Branch of the Russian Academy of Sciences \\ Laboratory of Optimal Control, \\ Lermontov str., 134 \\ Irkutsk, Russia \\ E-mail: tz@icc.ru
}

\begin{abstract}
The paper proposes a technology for a global extremum search, which makes it possible to estimate the probability of finding a solution in the optimal control problems. The idea of multistart based on proposed technique enables to create the computing technology which is not strongly depend on problem dimension and oriented to finding additional information about investigated object. The paper presents solutions of two nonconvex optimal control problems for power systems.
\end{abstract}

Keywords: Optimal control, power system, numerical method, computational technology.

\section{Introduction}

All algorithms that form the basis of familiar software for optimal control problems (OCP) are of a local nature; they are designed to search only the local extrema. This property for solving applied problems is inconvenient: there is never any certainty that there are no other solutions in the problem than the one already found. The problem of a global extremum search in OCP refers to another difficulty level of extreme problems; and at present, according to the authors, it is far from being resolved.

In the opinion of the authors, the hierarchy of nonlocal extremum problems, according to increasing complexity, is the following: P1 - search for several local extrema; P2 - search for all local and global extrema; P3 - finding a global extremum and proving that a global extremum is found.

We hold that, at present, there is no necessary theoretical basis for solving the problems P2 and P3 in OCP. For the problem P1, in contrast, there are several ideas proposed both in the theory of optimal control (for example, [1], [2]) and in the theory of finitedimensional optimization, based on the one that can try to build globalizing technologies for dynamic problems. The algorithm for solving the task P1 for OCP will be called globalizing algorithm.

Of all the approaches to the globalization of extremum search, the most simple and popular one is the idea of a multistart [3], [4]. A multistart is usually understood as the sequential or parallel multiple search for local minima from various initial approximations (for example, with a uniform or random grid). Usually, the following assumptions are made about the problem being solved: the finiteness of the number of local extrema, the remoteness of local extrema from each other, and the smoothness of the problem for the convenience of using local methods.

The multistart method provides numerous implementation options: a) the local extremum surroundings by the neighborhood - the achievement of these neighborhoods is considered as achieving the extremum (local extremum coverings); b) dynamic association of close-search trajectories (for example, cluster methods, nearest neighbor method); and other such methods. Some methods from the multistart family are considered among the most effective ones for finitedimensional global optimization problems. 
Among the other approaches, it is necessary to mention the methods of random search (RS): Monte Carlo method, uniform RS, uneven RS with a priori distribution, the method of stochastic coverage, annealing simulation methods), enumeration method of local extrema (for instance, heavy ball method, Branin's method), methods of integral representations (Chichinadze's method), and evolutionary algorithms ([5-8]).

Among the approaches developed in the optimal control theory, the methods of solving Bellman equation (Krotov-Bellman, Hamilton-Jacobi-Bellman methods) take a special place [9], [10]. They are, in particular, characteristics method and semidiscretization method.

An approach based on approximations of the reachable set of a controllable system [11] is also interesting.

The analysis of all the above approaches allows us to formulate the following conclusions:

- These approaches are applicable only to problems of optimal control of small dimensions.

- In all constructively realized cases, one can rely only on the achievement of a global extremum with unit probability.

- The least dependent on the dimension of the problem and practically the only tool for constructing globalizing algorithms for a wide class of problems continues to be multistart.

\section{Optimal control problem statement}

We consider optimal control problem with free right end

$$
\dot{x}=f(x, u, t), \quad t \in\left[t_{0}, t_{1}\right], x\left(t_{0}\right)=x^{0} .
$$

This problem is to find of control satisfying parallelepiped constraints $u(t) \in U$ and allows to achieve the minimum value of the terminal functional

$$
I(u)=\varphi\left(x\left(t_{1}\right)\right) \rightarrow \min .
$$

To solve problem (1)-(2), it is necessary to develop algorithms for searching several local extrema in OCP, which allow using existing software for local auxiliary problems and estimating the probability of finding a global minimum.

\section{Idea of computing technology}

To solve this problem, we developed a technology based on the multistart ideology. Multiple searches for local extremes are performed from various stochastic controls. Four algorithms are realized for generating pseudo-random start points (controls) based on various hypotheses about the class of admissible controls:

- table function,
- relay function,

- spline function,

- piecewise linear function.

Proposed technology include the criterion of convergence in the form of a modified statistical estimate of the probability of the existence of unidentified local extrema;

\subsection{Start points generating algorithms}

The algorithms for generating start points for the multistart scheme must meet the following criteria:

- the generated control must be pseudo-random,

- the generated controls must be lie within the parallelepiped constraints,

- the generated controls must be (in the limit) a dense set,

- the generated controls must belong to the class of piecewise continuous functions.

The segment of the independent variable (time) change is divided into $n u-1$ parts ( $n u-$ the number of sampling points), i.e. a uniform grid $T_{U}=\left\{t_{0}+i \cdot h, i=\overline{1, n u-1}\right\} \cup t_{1}$ is introduced, where $h$ is the sampling step. As a generator of quasi-random functions, the URAND algorithm (Universal RANDom number generator, [12]) is used, giving a uniformly distributed pseudo-random number from the interval [0,1], which will be denoted below $R$.

Algorithms SPLINE and SEVAL [12] are used to construct cubic spline functions.

\subsubsection{Algorithm for constructing random table functions}

Step 0. Set the algorithmic parameter $K_{U 0}-$ "growth rate in step".

Step 1. It is calculated $u_{1}=u l_{1}+R \cdot\left(u g_{1}-u l_{1}\right)$ in the first node of the grid $\left\{T_{U}\right\}_{1}=t_{0}$.

Step 2. In each grid node $T_{U}$ it is calculated

$$
\begin{aligned}
& \overline{u l}=u_{i-1}-K_{U 0} \cdot\left(u g_{i}-u l_{i}\right) \text { and } \\
& \overline{u g}=u_{i-1}+K_{U 0} \cdot\left(u g_{i}-u l_{i}\right), i=2, n u
\end{aligned}
$$

Step 3. The obtained values are projected onto an admissible parallelepiped:

if $\underline{\overline{u l}}<u l_{i}$ then $\overline{u l}=u l_{i}$,

if $\overline{u g}>u g_{i}$ then $\overline{u g}=u g_{i}$.

Step 4. Calculated $u_{i}=\overline{u l}+R \cdot(\overline{u g}-\overline{u l})$.

The algorithm is complete.

\subsubsection{Algorithm for constructing random relay functions}

Step 0. The algorithmic parameter $N_{T P}$ is set - the number of nodes of the "relay" grid.

Step 1. A random grid $T_{P}$ of $N_{T P}+2$ nodes is generated, such that $\left\{T_{P}\right\}_{1}=t_{0}$ and $\left\{T_{P}\right\}_{N T P+2}=t_{1}$. 
Step 2. For subintervals of a segment $\left[t_{0}, t_{1}\right]$ separated by a grid $T_{P}$, it is generated values $\overline{u t p}_{j}, \quad j=1, N_{T P}+1$, such that:

if $R<1 / 2$ then $\overline{u t p}_{j}=u l$ differently $\overline{u t p}_{j}=u g$.

Step 3. The resulting control is projected onto the original grid $T_{U}$.

The algorithm is complete.

\subsubsection{Algorithm for constructing random spline functions}

Step 0. The algorithmic parameter $N_{S P}$ is set - the number of nodes of a uniform "spline-grid"

Step 1. A uniform "spline-grid" $T_{S}$ is generated with a step $h_{S}=\left(t_{1}-t_{0}\right) /\left(N_{S P}-1\right)$,

such that $\left\{T_{S}\right\}_{1}=t_{0}$ and $\left\{T_{S}\right\}_{N S P+2}=t_{1}$.

Step 2. At the grid nodes $T_{S}$, random reference values $u s p_{\perp}$ are generated:

$\overline{u s p}_{j}=u l+R \cdot(u g-u l), \quad j=\overline{1, N_{S P}+2}$.

Step 3. Based on the grid $T_{S}$ and the generated reference values $\overline{u s p}_{j}$, a cubic spline function is constructed using the SPLINE and SEVAL algorithms.

Step 4. The values of the constructed spline function are projected onto the grid $T_{U}$.

Step 5. The resulting control is projected onto an allowable box $[u l, u g]$.

The algorithm is complete.

\subsubsection{Algorithm for constructing random piecewise-linear functions}

Step 0. The algorithmic parameter $N_{S}$ - the number of nodes in the uniform grid is set.

Spet 1. A uniform grid $T_{R}$ is generated with a step $h_{R}=\left(t_{1}-t_{0}\right) /\left(N_{S}-1\right)$,

such that $\left\{T_{R}\right\}_{1}=t_{0}$ and $\left\{T_{R}\right\}_{N S+2}=t_{1}$

Step 2. At the grid nodes $T_{R}$ nodes, random reference values are generated $\overline{u r}_{j}$ :

$$
\overline{u r}_{j}=u l+R \cdot(u g-u l), \quad j=\overline{1, N_{S}+2} .
$$

Step 3. Based on the $T_{R}$ grid and the generated reference values $\overline{u r}_{j}$, a piecewise linear function is constructed using linear interpolation.

Step 4. The values of the constructed piecewise-linear function are projected onto the grid $T_{U}$.

The algorithm is complete.

\subsection{The criterion of convergence is an estimate of the probability of finding a global extremum}

A statistical estimate of the existence probability of unidentified local extrema [4] is based on the probabilistic approach to the theoretical investigation of the random multistart-type algorithms. Modified for convenience of calculations, the formulas look as follows: $\gamma=\log _{10}\left(C_{E} \cdot \exp \left(-C_{S} / C_{E}\right)\right)$, where $C_{E}$ is the number of extremes found, $C_{S}$ - the number of multistarts, and $\gamma$ is the probability of non-existent extremes in the problem. The stopping criterion in the multistart algorithm can be as follows: $\gamma<\varepsilon_{G}$, where $\varepsilon_{G}$ is the algorithmic parameter. For calculations that do not require special guarantees of reliability, you can set the value $\varepsilon_{G}$ equal to $10-50$ ( $\varepsilon_{G}$ has a minus sign), at high requirements to the reliability of the global solution, $\varepsilon_{G}$ should be set about 300 .

Such a criterion of convergence cannot be held as fully adequate (its informativity depends strongly on the number of extrema in the problem; the sequence $\gamma$ is nonmonotonic on iterations), but other, more adequate criteria are yet to be developed.

\subsection{The effect of algorithmic parameters on the computational properties of the proposed technique}

Using the algorithms proposed in 3.1, the start controls are repeatedly generated, after which the extremum is searched using the original combination of reduced and conjugate gradients. The method stops depending on the value of the criterion given in paragraph 3.2.

The method of generating random table functions $\left(M_{G}=1\right)$ is controlled by a parameter $K_{U 0}$ that limits the rate of growth of a function at one discretization step. The allowed interval for the variation of the parameter $K_{U 0} \in[0.001,1.0]$, the recommended value is the default value $K_{U 0}=0.1$. For large values $K_{U 0}$ (of the order of unity) too oscillating starting controls are generated, which greatly slows down the operation of the local algorithm. For small values $K_{U 0}$ (of the order of 0.001), an unrepresentative sample of starting points can be generated.

The effectiveness of the method of relay functions generating $\left(M_{G}=2\right)$ is controlled by the parameter $N_{T P}$ - the maximum number of switching points in the start controls. The allowed interval for the variation of the parameter $N_{T P} \in[1,20]$, the recommended value is the default value $N_{T P}=5$. For small values $N_{T P}=1$, a loose set of starting controls is generated, large values may lead to a rise in the cost of local searching.

The method of generating spline functions ( $M_{G}=3$ ) seems to be the most stable to the variations of the parameter $N_{S P}$ controlling it (an allowable variation interval $N_{S P} \in[3,20]$, the recommended value by default $N_{S P}=10$ ). Good starting samples are generated for almost all allowable values of the parameter $N_{S P}$.

The generation of piecewise linear functions $\left(M_{G}=4\right)$ is controlled by a parameter $N_{S}$ (an 
allowable variation interval $N_{S} \in[3,20]$, the recommended value by default $N_{S}=10$ ). If the values are too large or too small $N_{S}$, the same problems can occur as with the generation of table functions.

The allowed interval for the variation of the parameter $\varepsilon_{G}$ is the probability of finding a global extremum $\varepsilon_{G} \in[-300.0,-10.0]$, the recommended value by default $\varepsilon_{G}=-20.0$. If the stopping criterion in the multi-start algorithm has worked $\left(\gamma<\varepsilon_{G}\right)$, then it means that, according to the evaluation technique used, a global extremum is likely to be found with probability $1-10^{\varepsilon_{G}}$. However, the accuracy of such an approach cannot be overestimated: the informativeness of the technique used depends significantly on the dimension of the problem and the number of local extremums [4], and the practice of applying the algorithm is still not large enough. It is more correct to consider $\gamma$ as an indirect characteristic of the quality of the multistart algorithm, the real value of which can be revealed by deep testing and long-term operation of the algorithm.

\subsection{The optimal control problem for power system}

Proposed in this paper computing technologies we used for solving nonconvex optimal control problem for power system [13], [14] in following statement

$$
\begin{gathered}
\dot{x}_{1}=x_{2} \\
\dot{x}_{2}=0.072-0.0968 x_{3}^{2}-0.0605 x_{3} \sin x_{1} \\
\dot{x}_{3}=0.232\left(1+u_{1}\right)-0.297 x_{3}+0.0919 \cos x_{1} \\
I_{0}(u)=t_{1}, \\
x(0)=(-0.0436,0,1.1), x\left(t_{1}\right)=(-0.4502,0,1.043), \\
t \in\left[0, t_{1}\right],\left|u_{1}(t)\right| \leq 1 .
\end{gathered}
$$

The results of computational experiments for the first problem are presented in Fig. $1-2$.

The minimum value of the objective functional is $I_{0}^{*}(u)=6.30012$. This result was obtained by $9 \mathrm{sec}$ of processor time (for a personal computer with characteristics: processor Intel Core $\mathrm{i} 5-2500 \mathrm{~K}$ and 16 $\mathrm{Gb}$ of RAM).

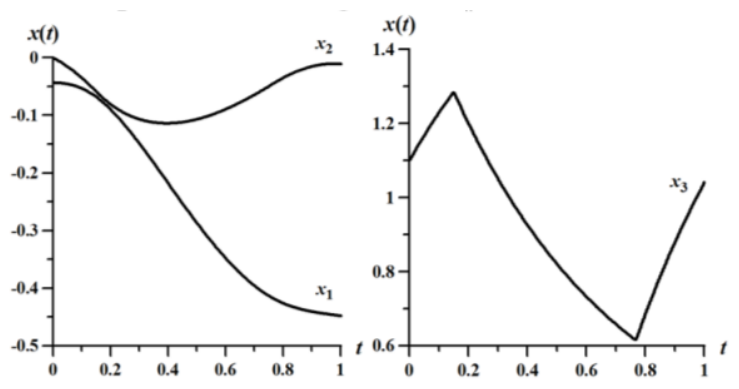

Fig. 1. Optimal trajectories in the control problem for power system.

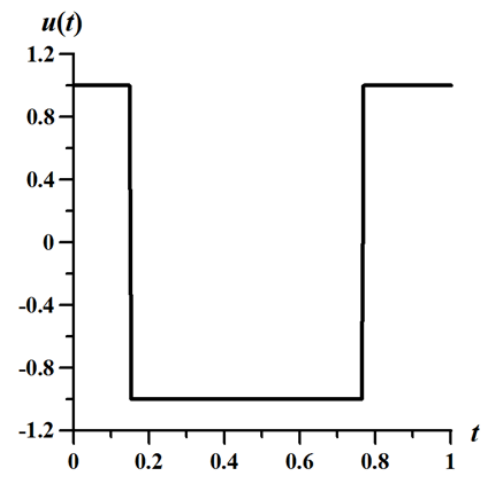

Fig. 2. Optimal control in the first problem.

\subsection{Optimization of the modes of the power grid with direct current elements}

When solving control problems for the development and functioning of electric power systems, special attention is paid to regime reliability and stability in emergency situations caused by sudden equipment failures, staff errors, etc.

The scientists of Melentiev Energy Systems Institute SB RAS were formulated the optimal control problem of the loading of DC-DC converters for the aggregated dynamic model of the Unified EPS of the USSR, represented by a two-node scheme^

$$
\begin{gathered}
\dot{x}_{1}=x_{3} \\
\dot{x}_{2}=x_{4} \\
\dot{x}_{3}=a_{1}\left(x_{5}-30 x_{3}-b_{1} \sin x_{1}-b_{2} \sin \left(x_{1}-x_{2}\right)-\right. \\
\left.-u_{1}(t-\tau)-15\right) \\
\dot{x}_{4}=a_{1}\left(x_{6}-30 x_{4}-b_{2} \sin \left(x_{2}-x_{1}\right)-u_{2}(t-\tau)-15\right) \\
\dot{x}_{5}=a_{2}\left(20-x_{5}-20 x_{3}\right) \\
\dot{x}_{6}=a_{2}\left(20-x_{6}-10 x_{4}\right)
\end{gathered}
$$

with initial conditions $x_{1}(0)=0.98511, \quad x_{2}(0)=$ $=0.5 x_{1}(0), x_{3}(0)=x_{4}(0)=0, x_{5}(0)=x_{6}(0)=20$. Here $x_{1}, x_{2}$ are relative angles of the voltage vector at the 2 and 3 stations; $x_{3}, x_{4}$ are rate of change $x_{1}$ and $x_{2}$; $x_{5}$ and $x_{6}$ are rated power of stations; $b_{1}, b_{2}$ are throughputs of direct current lines, $u_{1}, u_{2}$ are load converters in substations.

Under normal operating conditions, the system is in equilibrium while $u_{1}(t)=u_{2}(t)=5, \quad x_{3}(t)=x_{4}(t)=0$ The imbalance is formed during a disturbance, which is expressed in a change of the coefficients $b_{1}, b_{2}$. Phase variables are satisfy the following constraints

$$
\begin{gathered}
\left|x_{1}(t)\right| \leq \pi,\left|x_{1}(t)-x_{2}(t)\right| \leq \pi, \\
\left|x_{i}(t)\right| \leq 0.018, i=3,4 \quad x_{3}(t) \leq \arcsin (25 / 27), \\
\left|x_{3}-x_{4}\right| \leq \arcsin (25 / 27), t \in\left[t_{0}, t_{1}\right]
\end{gathered}
$$

Process control, performed with a delay of $\tau=0.02$ seconds, must also satisfy constraints $0 \leq u_{i}(t-\tau) \leq \min \{10,3 t\}, i=\overline{1,2}$. 
The problem is to transfer the system into normal mode after disturbance, i.e. search for controls that deliver a minimum of

$$
I_{0}(u)=\int_{t_{0}}^{t_{1}}\left(x_{3}^{2}(t)+x_{4}^{2}(t)\right) d t
$$

by 20 seconds for all restrictions.

The system, whose normal operating mode was carried out at $b_{1}=b_{2}=6$, was disturbed with $b_{2}=4$. The result of the solution is: $I_{0}\left(u^{*}\right)=2.7 \cdot 10^{-3}$ all constraints are satisfied with the required accuracy. Optimal control assumes redistribution of converter substations loads. The EPS regime stabilizes in 35 seconds.

\section{Conclusions}

The main problem of searching global extremum in OCP is the problem of guarantees achievement. The paper proposes a technology for a global extremum search, which makes it possible to estimate the probability of finding a solution in the problems of the class under consideration. The idea of multistart based on proposed technique enables to create the computing technology which is not strongly depend on problem dimension and oriented to finding additional information about investigated object. The paper presents solutions to two optimal control problems for power systems. The computational experiments carried out made it possible to demonstrate the effectiveness of the proposed computing technique.

\section{Acknowledgements}

The work is partially supported by Russian Foundation of Basic Research, grant No 17-07-00627.

\section{References}

1. V. A. Srochko, Iteration methods of optimal control problems solving (Moscow, FIZMATLIT, 2000).

2. A. S. Strekalovskii, Optimal control problems with terminal functionals represented as the difference of two convex functions, Comput. Math. Math. Phys. 47(11) (2007) 1788-1801.

3. A. Zhigljavsky, A. Zilinskas, Stochastic global optimization (Springer, New York, 2008).

4. A. Zilinskas, Global optimization. Axiomatic of stochastic models, algorithms and applications (Vilnius, Mokslas, 1986). (In Russian).

5. R. Brent, On the davidenko-branin methods for solving simultaneous nonlinear equations, IBM Journal of Research and Development, 16:434-436 (1972).

6. Ahmad M. Bakri Kasbah, Alessandro Astolfi and Dina Shona Laila, A modified Branin method, in Proc. 17th Int. Symposium on Mathematical Theory of Networks and Systems (Kyoto, Japan, 2006).

7. A. P. Karpenko, Population algorithms of global search searching optimization. Review of new and little-known algorithms, Information technologies, 7 (2012) 1-32. (In Russian)

8. A. I. Diveev, S. V. Konstantinov, Evolutionary algorithms for solving optimal control problem, Bulletin of the Peoples' Friendship University of Russia Series: Engineering studies, 18(2) (2017) 254-265.

9. V. F. Krotov, Global methods in optimal control theory (New York, Marcel Dekker Inc., 1996).

10. V. A. Dykhta, Weakly monotone solutions of the Hamilton-Jacobi inequality and optimality conditions with positional controls", Autom. Remote Control, 75:5 (2014), 829-844.

11. M. M. Hrustalev, Accurate description of attainability sets and global optimality conditions for dynamic systems, Automation and Remote Control, 5 (1988) 6270 .

12. J. Forsite, M. Malkolm, K. Mouler, Mechanical methods of mathematical calculus (Moscow, Mir, 1980). (In Russian).

13. A. Yu. Gornov, N. G. Kasimov, Yu. N. Kucherov, Model of optimal control of transient modes of EPS containing $D C$ elements (All-Union Scientific and Technical Conference "Creation of complexes of electrotechnical equipment for high-voltage, converter, high-current and semiconductor equipment", Moscow, 1989).

14. A. Yu. Gornov, Numerical technology for Optimal Control Problems (Novosibirsk, Nauka, 2009). (In Russian) 